\title{
Creencias sobre la enseñanza y uso de las tecnologías de la información y la comunicación (TIC) en docentes de educación superior
}

\author{
María L. Arancibia ${ }^{1}$ Julio Cabero² y Verónica Marín ${ }^{3}$ \\ (1) Centro de Innovación en Educación, CIEDU, INACAP, Brown Norte 290, Nuñoa-Chile. \\ (correo-e: marancibiam@inacap.cl) \\ (2) Facultad de Ciencias de la Educación, Universidad de Sevilla, c/ Jiménez Aranda ํㅜ 19, escalera 7 4으. ] \\ Sevilla-España. (correo-e: cabero@us.es) \\ (3) Facultad de Ciencias de la Educación, Universidad de Córdoba, Avda. San Alberto Magno s/n, 14004- \\ Córdoba-España. (correo-e: vmarin@uco.es)
}

Recibido Oct. 8, 2019; Aceptado Nov. 26, 2019; Versión final Ene. 30, 2020, Publicado Jun. 2020

\begin{abstract}
Resumen
El objetivo de esta investigación fue determinar una tipología de creencias en la enseñanza de los docentes, su relación al uso de Moodle, y posibles diferencias significativas entre perfiles y el uso de esta plataforma. Con una muestra representativa de 641 profesores de una universidad chilena, se analizó un cuestionario que midió las concepciones de enseñanza y el uso de tecnologías de la información y la comunicación (TIC) en el aula. Un análisis de clúster no jerárquicos de K-Medias generó dos perfiles de profesores: constructivista y conductista. Los constructivistas usaban la tecnología con mayor frecuencia, con apoyo de Moodle. Las magnitudes promedio de los índices para ambos fueron bajos, donde prácticamente todos no superaron la mediana de la escala utilizada. Esto nos llevó a confirmar que no existe una alineación entre creencias centradas en el estudiante y prácticas de aula de tecnología constructivista. Existen otras barreras como prácticas curriculares y una baja formación tecnológica y pedagógica.
\end{abstract}

\section{Beliefs on teaching and the use of information and communication technologies (ICT) by higher education professors}

\begin{abstract}
The aim of this study was to determine a typology of beliefs of professors' teaching and their relationships to the use of Moodle. This study also aimed to assess whether or not there were significant differences between profiles and the use of this platform. This study surveyed 641 professors from a Chilean university. The survey was designed to analyze and measure teaching concepts and information and communication technologies (ICT) usage in the classroom. A non-hierarchical K-Media cluster analysis generated two teacher profiles: constructivist and behavioral. Constructivists used technology with Moodle support more often than behavioral professors. The average magnitudes of the indices for constructivist and behavioral professors was low and the median did not exceed the scale used in most cases. This led us to confirm that there is no alignment between student-centered beliefs and constructivist technology classroom practices. Instead, there are other barriers such as curricular practices and low technological and pedagogical background.
\end{abstract}




\section{INTRODUCCIÓN}

Las barreras docentes se manifiestan en creencias, resistencias y actitudes negativas hacia las innovaciones pedagógicas, existe una persistencia en metodologías obsoletas de enseñanza y evaluación, una falta de percepción de la importancia de innovar al respecto, falta de capacidades técnicas en TICs. Estas son algunas de las resistencias docentes que aparecen en las investigaciones sobre experiencias de innovación con tecnología en enseñanza, junto con las dificultades para la apropiación de la innovación por parte de estos. Tal es el caso de los resultados rescatados de los estudios de Lašáková, Bajzíková y Dedze (2017), Mackeogh y Fox (2009) y por MINEDUC (2017) y Jara (2010) realizados en Chile para evaluar el programa ENLACES y su impacto en la docencia.

Así también, encontramos en el contexto chileno (MINEDUC, 2006; 2008), diferentes propuestas de estándares realizados por algunas instituciones, por ejemplo, la identificación de amplias dimensiones de estudio, alrededor de las cuales se han configurado las diferentes políticas de formación docente en TIC. Al mismo tiempo, encontramos en el sistema escolar estándares de mejoramiento profesional docente, entendido como aquellas habilidades que permiten a estos, dar continuidad a lo largo de la vida a procesos de aprendizaje con Tic. Así también, actualmente, está en funcionamiento un Centro de Innovación centrado principalmente en la educación escolar, cuyo objetivo se concentra en el mejoramiento de las prácticas educativas con apoyo de Tic en escuelas y liceos (MINEDUC, 2020). A nivel internacional, se ha avanzado en el Marco de Competencias Digitales, a través de la European Commission (INTEF, 2017), el cual se centra en que los docentes adquieran las competencias en gestión de la información, comunicación, colaboración y creación de contenido digital y resolución de problemas.

Frente a este contexto, las creencias que los docentes tienen sobre la enseñanza siguen siendo un factor que claramente determinan las estrategias que utilizan en ella, de ahí la significación de su investigación. Por otra parte, el propio término creencias tiene cierta confusión terminológica con otros, como el de concepciones, ideas previas, como lo han sugerido Coll y Remesal (2009). Estas creencias son construidas a partir de un ethos y de una cultura de una institución educativa y son aprendidas a través de los procesos de socialización y enculturación (Pajares, 1992, Ermert et al, 2017). En esta construcción de las creencias de la enseñanza confluyen dos grandes paradigmas, uno centrado en producir aprendizaje y fomentar en los estudiantes la construcción del conocimiento (constructivismo) y otro, cuyo punto de inflexión se encuentra en la transmisión de los conocimientos a los estudiantes, sin otorgar importancia a la reflexión y el análisis (paradigma tradicional).

Este último, dominante y hegemónico en la enseñanza (Estévez, Valdés, Arreola y Zavala, 2014). De todas formas, debemos reconocer que los términos creencias y concepciones son bastante ambiguos, y nosotros matizaremos el concepto de creencia siguiendo lo expuesto por García, Azcarate y Moreno (2006), quienes nos indican que las creencias vienen marcadas por las siguientes características: están asociadas a ideas a las ideas personales, influyen en el proceso de enseñanza-aprendizaje, tienen un valor afectivo, son un tipo de conocimiento, y se justifican sin rigor alguno. Por su parte las concepciones como señala Bohórquez (2018) "se describen como un subconjunto de creencias que son consciente" (p. 88). Por lo tanto, las creencias sobre la enseñanza de los docentes tienen mucho que ver con la integración que estos hagan de las tecnologías en su quehacer docente (Prestidge, 2012). Varias investigaciones señalan que los profesores usan la tecnología para desarrollar actividades que les ayudarán a acomodar sus propias perspectivas de enseñanza y aprendizaje (Admiraal ,2017; Orlando, 2013). Por lo tanto, los profesores con diferentes creencias pedagógicas integrarán la tecnología de manera diferente en sus aulas (Ertmer, Ottenbreit-Leftwich, Sadik, Sendurur y Sendurur, 2012.

Sobre la construcción de tipologías de docentes que integran tecnología en el aula, tenemos los aportes de Admiraal (2017) quien obtuvo cinco perfiles de profesores según la combinación de creencias sobre la enseñanza y niveles de pensamiento utilizados por Biggs y Tang (2011). Donde se concluyó sobre la necesidad de reflexión docente, trabajo colaborativo y generación de una cultura reflexiva y colaborativa entre los profesores. La evidencia empírica también señala que las creencias constructivistas de los profesores no siempre reflejan sus prácticas o su enseñanza con tecnología y se limita a prácticas convencionales de enseñanza (Mama y Hennessy, 2013). En otros estudios realizados por Orlando (2013) a través de un estudio longitudinal en Australia, se encontró una falta de relación entre la integración de la tecnología y las creencias de enseñanza centradas en el estudiante.

\section{OTROS ANTECEDENTES}

La configuración de la enseñanza puede variar dependiendo de las diferentes creencias que posean los profesores. Es ampliamente reconocido que las creencias educativas de los maestros son producto de su planificación, decisiones de instrucción y prácticas en el aula (Jimoyiannis y Komis, 2014). Algunos autores 
las denominan barreras de segundo orden y pueden tener un impacto crítico en la integración de la tecnología (Ertmer 2005). Estas creencias juegan un papel fundamental en la toma de decisiones sobre cómo utilizar una tecnología particular para apoyar el aprendizaje. Aunque los profesores reconocen la importancia de introducir las TIC en la educación, su uso es más bien limitado y marginal (Liu, Lin y Zhang, 2017), la mayoría de ellos continúan utilizando computadores para tareas como el procesamiento de textos, planificación de lecciones, elaboración y aplicación de pruebas u obtener información de internet (Jimoyiannis y Komis, 2014). Falta la aplicación de estrategias más significativas como las colaborativas (García-Chitiva y Suárez-Guerrero, 2019), muchas veces todo ello como consecuencia de su falta de formación (López, López y Prieto, 2018)

En profesores con creencias predominantemente constructivistas, las concepciones sobre la relación entre aprendizaje y enseñanza ocupan un lugar central, Asimismo son profesores que buscan crear un entorno de aprendizaje de significado, de negociación y fomento en la creación del conocimiento (Ertmert 2005). Este enfoque también denominado co-constructivista se basa en el diálogo, en el aprendizaje colaborativo y en la aplicación del conocimiento a problemas auténticos con apoyo de tecnología (Biggs, 2012). Los enfoques conductistas de la enseñanza apoyados con tecnología tienden a la transmisión y reproducción del conocimiento. Las investigaciones sobre aprendizaje constructivista han demostrado que los estudiantes aprenden mejor a través de la construcción de conocimiento donde las nuevas tecnologías deben servir para que el estudiante desarrolle autonomía, tomando un papel activo en solucionar problemas, comunicarse efectivamente, analizar información y diseñar soluciones (Hernández,2008;).

La tecnología se considera uno de los elementos importantes para el desarrollo de las habilidades de los estudiantes del siglo XXI, pues tienen un rol protagónico en la construcción del conocimiento. Es desde este tipo de transformaciones que se comienza a hablar de la sociedad del conocimiento, $y$, en este sentido, las formas de uso de las tecnologías se vuelven relevantes en tanto se comienzan a entender como medios que permiten acceder al conocimiento global En específico, en la educación superior está proliferando la utilización de diferentes plataformas virtuales, entre ellas la plataforma Moodle. La introducción de estas plataformas en educación va más allá de la gestión del aprendizaje, se trata de ir avanzando en la incorporación de metodologías activas, donde estos entornos virtuales de aprendizaje ofrecen mayores posibilidades pedagógicas. Algunos autores definen modelos de visibilidad de Moodle en función al rol que el profesor le concede tanto en sus guías como en las metodologías que utiliza en clases. Reconoce una enseñanza en la transmisión de conocimientos, una enseñanza donde se parte de la premisa de la participación activa como condición necesaria para el aprendizaje, donde el estudiante planifica, revisa y reflexiona, y donde el profesor es un facilitador el aprendizaje.

Los estudiantes resuelven problemas, producen y generan conocimiento (Sanchez, Sanchez y Ramos, 2012). Es así como la plataforma Moodle se configura en torno a lo que se denomina «pedagogía construccionista social» (es decir, conjuga aspectos del constructivismo donde el conocimiento se genera mediante mediación e interacción con el ambiente y del construccionismo (aprender haciendo), lo que permite el aprendizaje colaborativo. Se recomiendan la utilización de Moodle porque ofrece funcionalidades didácticas sofisticadas y ricas en opciones. Su flexibilidad, derivada de su estructura modular, es lo que garantiza dar soporte a cualquier estilo docente. (Sanchez, Sanchez y Ramos, 2012).

Se han realizado estudios y seguimientos sobre las potencialidades del Moodle como plataforma que apoya el proceso de enseñanza-aprendizaje (Sánchez, Sánchez y Ramos, 2012; González, 2017), entre ellas destaca la potencialidad de generar aprendizaje colaborativo, a través de la disponibilidad de recursos didácticos y la comunicación e intercambio de información entre estudiantes y docentes como principales factores facilitadores. Sin embargo, a pesar de sus potencialidades, diferentes investigaciones concluyen que efectivamente hay un uso mayor de las plataformas LMS (Learning Management System), pero no hay evidencia generalizada de un cambio en la práctica pedagógica (Liberona y Fuenzalida, 2014; CaberoAlmenara, Arancibia y Del Prete, 2019,

Por su parte, se han identificado limitaciones con respecto al uso de Moodle, que hacen referencia principalmente a factores humanos que a los límites de la herramienta en sí. En este sentido, las barreras tienen que ver más con la falta de uso y apropiación de la tecnología por parte de los docentes, que, con los límites propios de las distintas herramientas tecnológicas, por lo tanto, se diagnostica más bien un uso instrumental y funcional por parte de los profesores de la plataforma (González, 2017).

En consecuencia, se afirma que, el uso de Moodle depende de manera central de las creencias del profesor que tienden a influir en la integración tecnológica en su ejercicio docente, debido a que este es el responsable de crear contenidos, planificar las actividades didácticas y adoptar las diferentes herramientas tecnológicas (Gramp, 2013).

Por lo tanto, el éxito de LMS en cualquier institución comienza por la aceptación de esta por parte de los profesores y a la forma como se integra la tecnología al ejercicio docente. (Rivero, 2018) ), 
De ahí que el interés de este estudio sobre las creencias del profesorado en relación al uso de Tic, especialmente de la plataforma Moodle, se deba a que dichas creencias o concepciones intervienen en las situaciones de enseñanza-aprendizaje que estos utilizan, siviendo como marco de referencia y guías orientadoras de las acciones en sus prácticas educativas (Palos, 2017). Por tanto, este trabajo de naturaleza cuantitativa, tiene como objetivo proporcionar una tipología de profesores de educación superior, basada en sus creencias sobre la enseñanza y la integración de la plataforma Moodle en su práctica docente. Así también, busca contribuir con conocimiento a grupos de profesores para que puedan integrar la tecnología en su práctica pedagógica de forma eficaz.

\section{METODOLOGÍA}

El enfoque de la investigación es cuantitativo ya que tiene como objetivo determinar y explicar una realidad concreta a partir de mediciones numéricas, análisis estadístico, para así lograr determinar la relación entre las creencias en la enseñanza y el dominio técnico y didáctico de la plataforma Moodle. Los objetivos de este estudio son: 1) construir una tipología de profesores de educación superior basada en sus creencias sobre la enseñanza y la integración de la plataforma Moodle en su práctica docente; y 2) conocer la relación existente entre el uso de la plataforma Moodle y las creencias en la enseñanza de los profesores.

En cuanto a las preguntas de investigación, fueron las siguientes: 1) ¿Existen diferencias significativas entre los perfiles conductista y constructivista en el uso de la plataforma Moodle?; 2) ¿Existen diferencias significativas entre los perfiles por sexo y edad de los docentes que conforman los perfiles?; y 3) ¿Qué nivel de usabilidad tiene los docentes sobre el uso de herramienta, actividades y estrategias utilizadas con apoyo de la plataforma Moodle?

\section{Contexto de la investigación}

El Modelo Educativo Institucional de la Universidad Tecnológica de Chile, institución educativa donde se realizó este estudio, tiene como finalidad promover en los estudiantes el logro de resultados de aprendizaje definidos en términos de competencias, la pertinencia de las mismas en relación a la realidad del mundo del trabajo, las características y estilos de aprendizaje de sus estudiantes, los avances del conocimiento pedagógico respecto de la efectividad y pertinencia de las estrategias de enseñanza, y el progreso en las tecnologías de enseñanza-aprendizaje (MINEDUC, 2018 p.23).

Por ello, es importante determinar el uso y valoración que otorgan los profesores y estudiantes a las nuevas tecnologías de la información y comunicación (TIC) que están disponibles por la institución, así como aquellas que son propias del estudiante o del profesor y que son utilizadas en sus procesos académicos, enseñanza y aprendizaje respectivamente.

En el contexto de la educación superior chilena, históricamente, INACAP ha desarrollado y aplicado sistemas y tecnologías de información y comunicación, tanto en el ámbito de apoyo administrativo como académico. En este contexto, la figura del profesor es fundamental en los procesos formativos de esta universidad, dado que promueve un sistema de comunicación e interacción que generan sentido de pertenencia en los estudiantes, marcando un ritmo de trabajo y generando un andamiaje de apoyo para estos.

Por otro lado, el cuerpo docente lo constituye principalmente profesionales de distintos ámbitos de la ingeniería y otras áreas, un porcentaje mínimo, es profesor de formación inicial. El perfil referencial del docente se focaliza en su a) capacidad pedagógica para enseñar la disciplina, b) dominio de la disciplina c) experiencia y conocimiento del mundo productivo.

\section{Instrumento utilizado}

Se aplicó un cuestionario que fue desarrollado por el Centro de Innovación en Educación, CIEDU, de la Universidad Tecnológica de Chile INACAP en conjunto con la Universidad de Sevilla. Tuvo como finalidad recoger la visión de los profesores, así como las percepciones y oportunidades pedagógicas que perciben del uso de la plataforma Moodle que se implementó en la universidad como apoyo al trabajo pedagógico del profesor. Así también, se analizó la relación que existe entre el uso de esta plataforma y las creencias en la enseñanza de los profesores.

El cuestionario contó con un total de 21 preguntas de respuesta simple, así como de respuestas múltiples, divididos en siete secciones. La primera sección buscó caracterizar socio demográficamente a los y las docentes; una segunda sección intentó identificar las creencias de los docentes sobre su forma de enseñar, apartado que se basó en la escala de los autores De Vries, Van de Grift, y Jansen (2014). La tercera sección buscó identificar la frecuencia de uso de la plataforma; la cuarta, buscó determinar el dominio técnico y 
didáctico,) así como la frecuencia de uso de determinadas herramientas disponibles en la plataforma; la quinta, preguntó sobre las actividades y estrategias desarrolladas por el docente con apoyo de la plataforma; la sexta, consultó sobre los recursos de aprendizaje que los profesores usan en la plataforma; y por último, la séptima sección buscó indagar sobre las estrategias de trabajo de la asignatura en las que los docentes utilizan la plataforma.

\section{Muestra de estudio}

La aplicación del cuestionario se llevó a cabo durante el año 2017 de forma presencial a lo largo de todos campos de la universidad en estudio, por medio de una muestra representativa. La muestra se extrajo del total de docentes presentes (4.874), escogiendo una muestra proporcional estratificada por cada centro, buscando asegurar un error muestral de 3\%, con un nivel de confianza de $95 \%$. La muestra final alcanzó un total de 641 encuestas con respuestas válidas (tasa de respuesta promedio de 73\%). La muestra utilizada contó con un error muestral cercano al 3,6\%, con un nivel de confianza de $95 \%$. Si bien, esta muestra no corresponde al total previsto inicialmente, se asumió el aumento del error muestral de $0.3 \%$ debido al costobeneficio asociado con un aumento de la muestra, a través de una segunda aplicación de la encuesta.

La distribución de los encuestados en términos de sexo, promedio y tramos de edad estarían de acuerdo con las características de los y las docentes de toda la institución en cuestión (Ministerio de Educación, 2018), por lo que podemos asegurar que es representativa. De la muestra la distribución del sexo fueron $36 \%$ mujeres y $64 \%$ hombres. Su edad promedio era 42,37 años (SD $=11,07)$, que van desde los 21 a los 60 años y más años. La cantidad media de experiencia docente es de 11,45 años $(S D=9,02)$. El $60 \%$ obtuvo una alta calificación de educación profesional (licenciatura).

\section{Análisis factorial exploratorio (EFA)}

Primero, se operacionalizó las creencias de los profesores utilizando 14 ítems incluidos en el cuestionario antes descrito, que provienen de la adaptación realizada por De Vries et al. (2014) para medir las creencias con respecto a la enseñanza de los profesores. Los ítems se adaptaron en el cuestionario para utilizar una escala Likert de seis alternativas (1 Nada importante; 2 No tan importante; 3 Algo importante; 4 Importante; 5 Bastante importante; 6 Muy importante). Esto se realizó debido a que dicha escala permite una mayor flexibilidad en las respuestas de los profesores. Para analizar los ítems, se utilizó un Análisis Factorial Exploratorio (EFA), mediante el método de factores principales, reteniendo todos los factores que obtuvieran un valor propio (eigenvalue) mayor a 1. Adicionalmente, es importante mencionar que, dado que todas las variables consideradas fueron del tipo categóricas, fue necesario utilizar la forma Polychoric de la matriz de correlaciones en el análisis para obtener estimaciones robustas.

Luego se seleccionó solo aquellas variables con cargas factoriales mayores a 0.45 (esta medida corresponde a la correlación entre la variable original y el factor estimado). En caso de que existiesen variables complejas, esto es con cargas factoriales sobre el límite impuesto en más de un factor, se optó por dejar aquella donde la carga factorial fuese mayor, aunque dichas diferencias fuesen marginales. Lo anterior asegura que como mínimo un $20 \%$ de la varianza da cada variable sea explicada por cada factor seleccionado. Luego, para definir el tipo de rotación utilizada, se corroboró si la matriz de correlaciones entre los factores mostraba correlaciones altas (al no cumplirse esto, se optó por utilizar la rotación Varimax. Al igual que De Vries et al (2014) se obtuvo dos factores que agruparían los 14 ítems, que para efectos de esta investigación serán denominados: factor 1 de creencias conductistas y factor 2 de creencias constructivistas.

Una vez agrupados los ítems de la forma antes mencionada, se generaron dos índices que representarán creencias conductistas (Factor 1) y constructivistas (Factor 2) sobre la enseñanza de los profesores. Para esto, primero se comprobó la consistencia interna de las variables consideradas en cada índice. Las variables consideradas en el índice de creencias conductistas obtuvieron un Alpha de Cronbach igual a 0.89 y las variables consideradas en el índice de creencias constructivistas obtuvo un Alpha igual a 0.86. Lo que según los intervalos propuestos por George y Mallery (1995) para el Alpha de Cronbach corroboran que el instrumento de medición, en este caso las variables seleccionadas para cada índice serían excelentes, en cuanto a su consistencia interna. Los índices generados corresponden a las puntuaciones factoriales obtenidas a través de un promedio ponderado de las variables seleccionadas en cada factor. Los ponderadores (Tabla 1) fueron obtenidos mediante el porcentaje de la varianza de cada variable explicada por cada factor, dicho porcentaje fue re escalado para cada factor, de forma que los ponderadores obtenidos sumaran 1. Esta metodología corresponde a un método no refinado para estimar las puntuaciones de un índice (, estrategia que para este caso se muestra como una buena aproximación, ya que permite que las variables utilizadas no se repitan en distintos índices y que las puntuaciones finales tengan la misma escala de medición que los ítems originales, haciendo más simple y directa su interpretación. 
Tabla 1. Ponderaciones índice construidos

\begin{tabular}{|c|c|c|c|}
\hline & Preguntas & $\begin{array}{l}\text { Índice de creencias } \\
\text { conductistas }\end{array}$ & $\begin{array}{l}\text { Índice de creencias } \\
\text { constructivistas }\end{array}$ \\
\hline 1 & Transmitir el contenido de la materia a los/as alumnos/as. & $20 \%$ & \\
\hline 2 & Que el contenido de mis clases sea bueno. & $18 \%$ & \\
\hline 3 & Los alumnos/as adquieran conocimiento. & $18 \%$ & \\
\hline 4 & Los/las alumnos/as realmente escuchen lo que les digo. & $17 \%$ & \\
\hline 5 & Haya orden y disciplina durante la clase. & $10 \%$ & \\
\hline 6 & Los/las alumnos/as aprendan el contenido de la materia que enseño. & $18 \%$ & \\
\hline 7 & $\begin{array}{l}\text { Los/las alumnos/as comprendan como lograr un mejor aprendizaje de } \\
\text { la materia que enseño. }\end{array}$ & & $11 \%$ \\
\hline 8 & $\begin{array}{l}\text { Los/las alumnos/as aprendan a solucionar en forma autónoma los } \\
\text { problemas relacionados con la materia que enseño. }\end{array}$ & & $11 \%$ \\
\hline 9 & $\begin{array}{l}\text { Los/las alumnos/as, en la medida en que sea relevante, aprendan en } \\
\text { forma cooperativa en grupos de trabajo. }\end{array}$ & & $14 \%$ \\
\hline 10 & Los/las alumnas desarrollen sus capacidades y competencias. & & $15 \%$ \\
\hline 11 & $\begin{array}{l}\text { Establecer comunicación con el conocimiento y experiencias propias } \\
\text { de los/las alumnos/as. }\end{array}$ & & $15 \%$ \\
\hline 12 & $\begin{array}{l}\text { Considerar las diferencias en aptitudes e intereses entre los/las } \\
\text { alumnos/as. }\end{array}$ & & $15 \%$ \\
\hline 13 & $\begin{array}{l}\text { Integrar en mis clases los más recientes avances en el campo de mi } \\
\text { disciplina curricular. }\end{array}$ & & $10 \%$ \\
\hline 14 & Los/las alumnos/as trabajen activamente con el material que enseño. & & $10 \%$ \\
\hline
\end{tabular}

\section{Análisis multivariable}

Para analizar la relación que existe entre el uso de la tecnología en el aula con el tipo de creencia sobre el aprendizaje que predomina en los docentes, se generaron perfiles de docentes. De esta forma se buscó asociar la predominancia de una determinada creencia con el uso e integración de la plataforma tecnológica Moodle. Lo anterior se realizó generando tres perfiles de profesores basados en los dos índices de creencias construidos. Para generarlos, se utilizó un análisis de clúster no jerárquicos de K-Medias (Este método únicamente se puede aplicar a atributos numéricos cuya función objetivo, es la suma de los cuadrados de los errores entre los puntos y sus centroides respectivos, es igual a la varianza total dentro del propio clúster (

\section{RESULTADOS}

Los promedios para cada perfil generado se muestran en la Tabla 2, en esta muestra cerca de un $81 \%$ de los profesores encuestados tendrían una dominancia por una creencia en particular, dominancia que está justificada debido a que, para el Perfil 1 como para el Perfil 2 las diferencias entre las creencias observadas fueron significativas $(p<.01)$. En la tabla 1, se muestran las ponderaciones arrojadas por índice construidos. El test Kruskall-Wallace mostró que los índices generados varían significativamente a través de los tres perfiles generados. Más aún, el test de Mann-Whitney mostró que existirían diferencias significativas $(p<.01)$ entre cada clúster para ambas creencias. El denominado Perfil 1 agrupó a docentes para quienes las creencias conductistas tendrían predominancia, así estos serían docentes a los que mayormente les preocupa que el contenido de sus clases sea bueno, que los alumnos realmente escuchen lo que ellos dicen y que sus alumnos puedan aprender el contenido de la materia que enseñan, entre otras ideas. Perfil que será denominado conductista en adelante. Por el contrario, el Perfil 2 reúne a los docentes que mostraron dominancia por las ideas constructivistas, de esta manera ideas como "Los/las alumnos/as, en la mediad que sea relevante, aprendan en forma cooperativa en grupos de trabajo", "los/las alumnos/as desarrollen sus capacidades y competencias" y "establecer comunicación con el conocimiento y experiencias propias de los/las alumnos/as", serían muy relevantes para en su forma de enseñar. Este perfil será denominado constructivista en adelante.

Tabla 2. Promedio índices generados para cada perfil estimado

\begin{tabular}{|l|c|c|c|}
\hline & Perfil 1 & Perfil 2 & Perfil 3 \\
\hline Creencias Conductistas & 5.73 & 5.09 & 6 \\
\hline & $(0.41)$ & $(0.77)$ & $(0.00)$ \\
\hline Creencias Constructivistas & 5.35 & 5.68 & 6 \\
\hline & $(0.51)$ & $(0.48)$ & $(0.00)$ \\
\hline Observaciones & 274 & 242 & 125 \\
\hline
\end{tabular}


Finalmente, cerca de un $20 \%$ de los docentes encuestados no mostraron ninguna dominancia por alguna creencia, todos estos docentes se agrupan en el Perfil 3, al que llamaremos perfil de creencias mixtas. Con respecto a los rangos etarios de los docentes y los perfiles definidos, se encontró que, para el perfil constructivista, las creencias conductistas presentarían diferencias significativas $(p<.05)$, a través de los tramos etarios, mostrando una reducción de casi un punto entre el primer tramo hasta el último. Este hallazgo podría interpretarse como una reducción de la influencia que las creencias conductistas tendrían sobre el comportamiento de los profesores a través de los años.

\section{Relación entre creencias y tecnología}

Para analizar la dominancia de una determinada creencia con el uso e integración de la plataforma Moodle, se exploró la relación de los perfiles de creencias generados con las distintas dimensiones consultadas en la encuesta a docentes sobre esta plataforma. Específicamente, se analizó cuál es la relación entre los perfiles construidos con los índices de dominio técnico, manejo didáctico y frecuencia de uso de las herramientas de la plataforma, el índice de actividades realizadas con la plataforma y, finalmente, se analizó la relación con los índices de materiales tecnológicos utilizados por los profesores. Los resultados que se observan en la Tabla 3 muestran que en general, las herramientas disponibles en la plataforma Moodle tienen una frecuencia de uso bastante baja, así mismo, el dominio técnico de estas como el manejo didáctico de las mismas, si bien es más elevado que la frecuencia de uso no superaría la mediana de la escala utilizada (4.5 puntos).

Tabla 3. Promedio índices generados para cada dimensión considerada

\begin{tabular}{|l|c|c|c|c|c|}
\hline Índice & Observaciones & Media & DS & Min & Max \\
\hline Índice dominio técnico de herramientas. & 641 & 4.03 & 2.97 & 0 & 10 \\
\hline Índice manejo didáctico de herramientas. & 640 & 3.55 & 2.74 & 0 & 10 \\
\hline Índice frecuencia de uso de herramientas. & 641 & 2.42 & 1.98 & 0 & 10 \\
\hline Índice frecuencia de uso de actividades y estrategias. & 640 & 3.81 & 2.40 & 0 & 10 \\
\hline Índice de materiales tecnológicos de mayor sofisticación utilizados. & 639 & 2.21 & 2.04 & 0 & 10 \\
\hline Índice de materiales de uso común utilizados. & 640 & 6.84 & 2.66 & 0 & 10 \\
\hline
\end{tabular}

Al analizar con mayor profundidad los resultados descriptivos en la tabla 4, es posible observar una realidad aún más compleja donde herramientas como correo electrónico, foros, entrega de tareas y envío de videos, son las únicas que logran superar la mediana de la escala utilizada, esto en relación con el dominio técnico declarado por los mismos docentes. En cambio, si observamos la frecuencia de uso de las mismas herramientas, solo el correo electrónico y entrega de tareas superan la mediana de la escala utilizada. Aún más, 7 de 11 de las herramientas disponibles en la plataforma Moodle de la institución prácticamente no son utilizadas por los docentes (no alcanzan a superar 2.5 puntos), ni tampoco presentarían un dominio técnico 0 un uso didáctico por parte de los docentes.

Con respecto a las actividades y estrategias desarrolladas con el apoyo de la plataforma, nuevamente el índice estimado no sobrepasaría la mediana de la escala utilizada, por lo que el uso del conjunto de estrategias consultadas en la encuesta sería bajo. Al observar las descriptivas de los ítems considerados en dicho índice, es posible observar que la retroalimentación a los estudiantes, la realización de trabajos colaborativos y el uso de rúbricas de evaluación visibles para los alumnos, son las principales actividades y estrategias implementadas por los docentes a través de la plataforma Moodle. Por otro lado, la realización de debates, las simulaciones, la entrega de premios y actividades de reflexión para los estudiantes se desarrollarían con menor frecuencia.

Con respecto a los materiales utilizados, se observa que la plataforma es utilizada principalmente para la entrega de materiales de uso común, tales como guías, apuntes, presentaciones y artículos de lectura. En cambio, para aquellos elementos de mayor sofisticación que provee la plataforma, su frecuencia de uso sería extremadamente baja. En este ámbito se encontrarían materiales multimedia interactivos, audios, ejercicios de auto evaluación, exámenes en línea, y el uso de simuladores y/o laboratorios virtuales. Una vez mostrado que el dominio, frecuencia, e implementación de herramientas tecnológicas, así como las actividades y materiales que pueden ponerse a disposición de los estudiantes es, en general bajo. En la Tabla 5, se muestra si existe alguna diferencia entre estas dimensiones y los perfiles de creencias sobre la enseñanza creados en este estudio. La Tabla 5 muestra que en general todos los índices tienen una mayor frecuencia para el perfil constructivista que para el perfil conductista, resultado que no es tan claro al observar el perfil de creencias mixto, donde en algunos índices domina uno u otro. 
Tabla 4 Estadísticas descriptivas sobre dominio técnico, dominio didáctico y frecuencia de uso de herramientas Moodle

\begin{tabular}{|c|c|c|c|c|c|}
\hline Variable & $\begin{array}{l}\text { Dominio } \\
\text { Técnico }\end{array}$ & $\begin{array}{l}\text { Dominio } \\
\text { Didáctico }\end{array}$ & $\begin{array}{c}\text { Frecuencia } \\
\text { de uso }\end{array}$ & Min & Max \\
\hline \multirow{2}{*}{ Correo electrónico } & 8.02 & 7.11 & 8.28 & 0 & 10 \\
\hline & $(3.44)$ & (3.62) & (3.04) & & \\
\hline \multirow[t]{2}{*}{ Foros } & 4.76 & 4.15 & 3.09 & 0 & 10 \\
\hline & $(4.08)$ & $(3.81)$ & $(3.34)$ & & \\
\hline \multirow[t]{2}{*}{ Blog } & 3.05 & 2.60 & 1.57 & 0 & 10 \\
\hline & (3.62) & (3.28) & (2.33) & & \\
\hline \multirow[t]{2}{*}{ Wiki } & 3.06 & 2.65 & 1.63 & 0 & 10 \\
\hline & (3.59) & (3.31) & $(2.40)$ & & \\
\hline \multirow[t]{2}{*}{ Tareas (entrega de archivos para calificación o retroalimentación) } & 6.27 & 6.01 & 5.63 & 0 & 10 \\
\hline & $(4.03)$ & $(4.00)$ & $(3.93)$ & & \\
\hline \multirow[t]{2}{*}{ Glosario } & 3.61 & 3.00 & 2.14 & 0 & 10 \\
\hline & $(3.85)$ & $(3.53)$ & $(2.96)$ & & \\
\hline \multirow[t]{2}{*}{ Videos (YouTube, Vimeo, etc.) } & 5.35 & 4.94 & 4.15 & 0 & 10 \\
\hline & $(4.16)$ & $(4.02)$ & $(3.78)$ & & \\
\hline \multirow[t]{2}{*}{ Videoconferencia/Audio conferencia } & 2.86 & 2.45 & 1.53 & 0 & 10 \\
\hline & $(3.57)$ & $(3.24)$ & $(2.40)$ & & \\
\hline \multirow[t]{2}{*}{ Cuestionarios online } & 3.54 & 3.20 & 2.15 & 0 & 10 \\
\hline & $(3.91)$ & $(3.73)$ & $(3.01)$ & & \\
\hline \multirow[t]{2}{*}{ Co-evaluaciones (tú evalúas a tus compañeros y ellos a ti) } & 2.98 & 2.76 & 1.75 & 0 & 10 \\
\hline & $(3.62)$ & $(3.47)$ & $(2.64)$ & & \\
\hline \multirow[t]{2}{*}{ Consultas o encuestas } & 3.60 & 3.26 & 2.40 & 0 & 10 \\
\hline & $(3.89)$ & $(3.69)$ & $(3.13)$ & & \\
\hline Observaciones & 641 & 640 & 641 & & \\
\hline
\end{tabular}

En este sentido, al comparar los grupos a través del test Kruskall-Wallace fue posible distinguir que existían diferencias significativas entre los perfiles de creencias solo para los índices: frecuencia de uso de herramientas $(p<.05)$, frecuencia de uso de actividades y estrategias $(p<.01)$, así como para el índice de materiales de uso común utilizados en la plataforma Moodle $(p<.1)$. El resto de los índices construidos no mostraron variaciones significativas entre los tres perfiles de creencias, por lo que no es posible determinar con claridad, si uno de los perfiles ya sea conductista, constructivista o mixto muestra una dominancia clara sobre los otros.

Tabla 5: Promedio índices generados para cada dimensión considerada con respecto al perfil de creencias

\begin{tabular}{|c|c|c|c|}
\hline Índice & $\begin{array}{c}\text { Perfil } \\
\text { Conductista }\end{array}$ & $\begin{array}{c}\text { Perfil } \\
\text { Constructivista }\end{array}$ & $\begin{array}{l}\text { Perfil } \\
\text { Mixto }\end{array}$ \\
\hline \multirow[t]{2}{*}{ Índice dominio técnico de herramientas } & 3.95 & 4.17 & 3.93 \\
\hline & $(2.81)$ & $(2.95)$ & (3.32) \\
\hline \multirow[t]{2}{*}{ Índice manejo didáctico de herramientas } & 3.38 & 3.63 & 3.76 \\
\hline & $(2.55)$ & $(2.66)$ & (3.27) \\
\hline \multirow[t]{2}{*}{ Índice frecuencia de uso de herramientas } & 2.14 & 2.49 & 2.91 \\
\hline & $(1.70)$ & $(1.93)$ & $(2.50)$ \\
\hline \multirow[t]{2}{*}{ Índice frecuencia de uso de actividades y estrategias } & 3.37 & 3.78 & 4.81 \\
\hline & $(2.25)$ & $(2.20)$ & $(2.77)$ \\
\hline \multirow[t]{2}{*}{ Índice de uso de materiales tecnológicos de mayor sofisticación utilizados } & 2.03 & 2.10 & 2.80 \\
\hline & $(1.87)$ & $(1.83)$ & $(2.63)$ \\
\hline \multirow[t]{2}{*}{ Índice de uso de materiales de uso común utilizados } & 6.62 & 6.85 & 7.29 \\
\hline & $(2.81)$ & $(2.52)$ & $(2.55)$ \\
\hline Observaciones & 274 & 242 & 125 \\
\hline
\end{tabular}

El análisis realizado no permite identificar si los perfiles relevantes (conductista y constructivista) muestran diferencias significativas, entre sí, en el manejo de la plataforma Moodle. Por esta razón, para determinar si existen diferencias significativas, se utilizó el test Mann-Whitney. Los resultados muestran que solo para los índices frecuencia de uso de herramientas $(p<.05)$ y frecuencia de uso de actividades y estrategias $(p<.05)$ existirían diferencias significativas entre los perfiles conductistas y constructivistas, siendo estos últimos quienes utilizarían mayormente la plataforma Moodle. Así, es posible afirmar que los docentes con perfiles más constructivistas serían quienes están utilizando con mayor frecuencia la plataforma y sus herramientas, y también serían ellos quienes aplicarían con mayor frecuencia actividades utilizando esta plataforma. De 
todas formas, es importante relevar y tener en consideración que las magnitudes promedio de los índices para ambos perfiles son bastante bajas, donde prácticamente todos no superarían la mediana de la escala utilizada.

Con respecto a las diferencias por sexo, se observa que para las docentes mujeres ningún índice presentaría diferencias significativas entre los perfiles conductistas y constructivistas. Así, para las mujeres, la posición frente a la plataforma Moodle se muestra independiente de sus creencias con respecto a la enseñanza. En cambio, para los hombres se observó diferencias significativas, siempre a favor de los docentes con perfil constructivista, para los índices de dominio técnico $(p<.1)$, manejo didáctico $(p<.05)$ y frecuencia de uso de herramientas $(p<.05)$, y también para el índice de frecuencia de uso de actividades y estrategias en la plataforma Moodle $(p<.05)$. Este resultado releva que los docentes hombres con perfiles conductistas, para quienes dominan las ideas centradas en el contenido de sus clases y en la obediencia de los alumnos, serían quienes encuentran menor utilidad a la plataforma y, además, podrían ser quienes tienen menores conocimientos sobre la misma y, por ende, ser quienes muestran un menor interés por apoyar sus clases con esta plataforma.

Finalmente, con respecto a la edad de los docentes encuestados, se observó que, para todos los docentes mayores de 41 años, ninguno de los índices generados tendría diferencias estadísticamente significativas entre los perfiles de creencias sobre la educación construidos. Sin embargo, para los docentes menores de 40 años, se observaron diferencias significativas, a favor del perfil constructivista, para el índice de frecuencia de uso de herramientas de la plataforma $(p<.1)$ y para el índice sobre la frecuencia en que han utilizado la plataforma para realizar actividades $(p<.05)$, tales como retroalimentar a los alumnos, realizar trabajos, utilizar rúbricas de evaluación, realizar ensayos, entre otras. Con esto, es posible mencionar que, para los docentes con más experiencia no existirían diferencias significativas en sus percepciones sobre la tecnología y sus creencias sobre la enseñanza, a diferencia de lo que ocurriría entre los docentes de menor edad donde sí se observó una diferencia entre sus creencias sobre la enseñanza y el índice sobre frecuencia de uso de las herramientas de la plataforma, y en el índice sobre actividades y estrategias que realizan utilizando la plataforma Moodle.

\section{DISCUSIÓN}

Hay evidencia significativa de que los profesores pueden y tienen simultáneamente creencias pedagógicas aparentemente contradictorias, alternando, por ejemplo, prácticas de enseñanza centradas tanto en el estudiante como en el contenido. A este perfil se le denominó conductista, debido a que sus creencias se centran tanto en la trasmisión de contenidos como en el creer que el éxito académico no depende de la forma que ellos enseñan, sino que depende exclusivamente de la motivación y conocimiento previo que puedan tener sus estudiantes (Biggs y Tang, 2011; Admiraal et al, 2017). Cuyo punto de inflexión se encuentra en la transmisión de los conocimientos a los estudiantes, y que ha sido dominante y hegemónico en la enseñanza (Estévez et al., 2014)

Por el contrario, el Perfil 2 o denominado constructivista se caracteriza por profesores que mostraron predominancia por creencias constructivistas de la enseñanza, centrados en que los estudiantes aprendan de manera colaborativa y en el desarrollo de competencias, a través de evaluaciones auténticas. En los profesores con esta predominancia, las concepciones sobre la relación entre aprendizaje y enseñanza ocupan un lugar central, con una preocupación mayor por crear entornos de aprendizaje significativo y de negociación, para el fomento y desarrollo del conocimiento (Ertmert 2005, Ermert et al, 2017). Este enfoque también denominado co-constructivista se basa en el diálogo, en el aprendizaje colaborativo y en la aplicación del conocimiento a problemas auténticos con apoyo de la tecnología (Biggs, 2012; Ermert 2005).

Se puede afirmar también, que la frecuencia de uso de la plataforma que en general fue bajo porque prácticamente no son utilizadas por los docentes (no alcanzó a superar 2.5 puntos), se realiza con herramientas poco complejas y que tienen una función más bien de repositorio como lo son el correo electrónico y la entrega de tareas. Está claro a su vez que, la frecuencia no determina el uso pedagógico de la plataforma (Fariña et al, 2013). Según estudios, las barreras de una usabilidad menos instrumental tienen que ver más con la falta de uso y apropiación de la tecnología por parte de los docentes, que con los límites propios de las distintas herramientas tecnológicas (González, 2017). Sobre los materiales utilizados, se ve que la plataforma está siendo utilizada principalmente para hacer entrega de guías, apuntes, presentaciones y artículos de lectura. En cambio, para aquellos elementos de mayor sofisticación que provee la plataforma Moodle, su frecuencia de uso es extremadamente baja (materiales multimedia interactivos, audios, ejercicios de auto evaluación, exámenes en línea, y el uso de simuladores y/o laboratorios virtuales), lo que se confirma con estudios que afirman el bajo uso de las potencialidades comunicativas y colaborativas, como de herramientas y recursos de la plataforma Moodle (Fariña et al, 2013). 
Por lo tanto, las creencias del docente sobre el aprendizaje y la enseñanza son factores críticos en el momento de integrar las TIC en el aula (Orlando, 2009), demuestra que el uso de la tecnología no asegura la transformación de las prácticas pedagógicas, ya que la práctica es un reflejo de las creencias del docente (Así también es importante resaltar que las razones para la disparidad entre las prácticas y las creencias, se relacionan con las limitaciones o barreras externas impuestas a los docentes mediante, por ejemplo, prácticas curriculares o de evaluación predeterminadas (Ermert, 2015) o por el grado de aceptación de la tecnología del LMS, que influiría en el profesor en relación con su actitud sobre uso y utilidad de la tecnología con fines pedagógicos (Admiraal et al., 2017; Arancibia, Halal y Romero, 2017).

En los resultados descriptivos, es posible observar una realidad aún más compleja donde solo las herramientas de correo electrónico, foros, entrega de tareas y envío de videos, serían las únicas que logran superar la mediana de la escala utilizada, en favor del perfil constructivista. Aún más, 7 de 11 de las herramientas disponibles en la plataforma Moodle prácticamente no son utilizadas por los profesores independiente de su predominancia y de su manera de enseñar; estos resultados coinciden con estudios que señalan que en muchos casos esta integración es de carácter formal, y se limita al uso del espacio virtual como repositorio de contenido (Pérez-Berenguer y García-Molina, 2016).

Si bien, estos resultados confirman que no necesariamente existe una alineación entre creencias y uso de la tecnología, será importante realizar una profundización cualitativa en esta área, apoyadas con observaciones de aula y entrevistas a profesores sobre sus prácticas pedagógicas con apoyo de TIC. Considerando en dicho estudio, teorías que se ajustan al uso pedagógico y didáctico de los LMS, como la denominada teoría de la actividad (Hashim y Jones, 2017) donde la clase o interacción presencial y el apoyo en línea, genera una confluencia tecno-pedagógica (Vásquez, 2017), que tiene como objetivo desarrollar grados crecientes de autonomía en el estudiante y donde el profesor es quien se observa como un mediador, facilitador y diseñador de las situaciones de aprendizaje.

Por otra parte, los estudios existentes han confirmado que existen diferencias significativas de género en el uso de la tecnología (Yong-Mi, 2010) como se demostró en esta investigación donde los profesores hombres con perfiles conductistas mostraron un menor interés por apoyar sus clases con la plataforma Moodle. Mientras que el perfil constructivista, evidenció disminuir sus creencias conductistas $(p<.05)$ a través de los tramos etarios. Este hallazgo podría interpretarse como una reducción de la influencia que las creencias conductistas tendrían sobre el comportamiento de los profesores a través de los años, atribuyéndosele a la experiencia y los años de formación de profesores más expertos (Admiraal et al., 2017). Por lo tanto, una identificación y un análisis de las creencias basado en estos paradigmas de aprendizaje constituyen un avance para identificar aspectos fundamentales para la formación docente, en ámbitos relacionados con los procesos de enseñanza-aprendizaje. Así también para promover el aprendizaje del estudiante a través de la participación colaborativa en tareas multidisciplinarias desafiantes y auténticas, y con apoyo de la tecnología.

Finalmente, en esta investigación asumimos una serie de limitaciones, entre las que podemos señalar el haber sido realizada en una Universidad privada y con una fuerte orientación hacia el terreno tecnólogo y profesional, y la necesidad de utilizar otros instrumentos de recogida de información como bien pudiera ser la entrevista en profundidad y la observación no participante de las interacciones y acciones realizadas por los docentes dentro de su aula virtual. Dichas limitaciones nos sugieren también líneas futuras de investigación como son el replicar la investigación en universidades públicas, discriminar las creencias de los profesores en función de su experiencia profesional y conceptual, y la incorporación a la investigación de otro tipo de instrumentos que nos permitan perfilar el tipo de creencia del docente.

\section{CONCLUSIONES}

De este estudio, los antecedentes, la discusión y análisis, se desprenden las siguientes conclusiones principales:

1) Más del 80 por ciento de los profesores tendrían una predominancia o por creencias centradas en el docente (transmisivas) o constructivistas (centradas en el estudiante), lo que permitió la creación de dos tipos de perfiles docentes; uno conductista, donde la enseñanza está centra en el profesor y uno constructivista, centrado en los aprendizajes del estudiante.

2) La frecuencia de uso y nivel de dominio pedagógico en la implementación de herramientas tecnológicas, así como las actividades y materiales que pueden ponerse a disposición de los estudiantes es en general bajo, lo que coincide con la literatura analizada.

3) Es posible afirmar que los docentes con perfiles más constructivistas serían quienes están utilizando con mayor frecuencia la plataforma y sus herramientas, y también serían quienes realizan más actividades de 
aprendizaje en la plataforma Moodle. De todas formas, es importante relevar y tener en consideración que las magnitudes promedio de los índices para ambos perfiles son bajas, donde prácticamente todos no superarían la mediana de la escala utilizada.

4) Los docentes hombres con perfiles conductistas serían quienes encuentran menor utilidad a la plataforma y es posible que presenten barreas y resistencias al uso de las TIC en su práctica educativa.

\section{REFERENCIAS}

Admiraal, W., Louws, M., y otros 10 autores, Teachers in School-based Technology Innovations: A Typology of Theirs Beliefs on Teaching and Technology, doi: https://doi.org/10.1016/j.compedu.2017.06.013, Computers \& Education 114, 57-68 (2017).

Arancibia, ML., Halal, C., y Romero, R., Valoración y Barreras en la Integración del e-portafolio en el Proceso de Práctica Inicial por Parte de Docentes y Estudiantes de Educación Superior, doi: http://dx.doi.org/10.12795/pixelbit.2017.i51.10 Píxel-Bit. Revista de Medios y Educación, (51), 151-163, (2017).

Biggs, J. B., y Tang, C. S., Teaching for Quality Learning at University: What the Student does (4th ed.), McGraw-Hill Education, Maidenhead, United Kingdom, (2011).

Biggs, J., What the Student does: Teaching for Enhanced Learning, doi: https://doi.org/10.1080/0729436990180105 Higher Education Research \& Development, 31(1), 39e55, (2012).

Bohórquez, L.A. Factores que Apoyan o Limitan los Cambios de Concepciones de los Estudiantes para Profesor de Matemática sobre la Gestión del Proceso de Enseñanza-Aprendizaje. AlEM - Avances de Investigación en Educación Matemática, 13, 85 - 10. (2018).

Cabero-Almenara, J., Arancibia, M. L., y Del Prete, A., Technical and Didactic Knowledge of the Moodle LMS in Higher Education. Beyond Functional Use, doi:10.7821/naer.2019.1.327. Journal of New Approaches in Educational Research 8(1), PP. 25-33. (2019).

Coll, C., y Remesal, A., Concepciones del Profesorado de Matemáticas acerca de las Funciones de la Evaluación del Aprendizaje en la Educación Obligatoria, doi: 10.1174/021037009788964187. Infancia y Aprendizaje, 32:3, 391-404, (2009)

De Vries, S., Van de Grift, W., y Jansen, E., How Teachers' Beliefs About Learning and Teaching Relate to Their Continuing Professional Development, doi. https://doi.org/10.1080/13540602.2013.848521. Teachers and Teaching, 20(3), 338-357, (2014).

Jara, I., Políticas de Informática Educativa para las Escuelas. Elementos Clave para su Diseño. En Bilbao, A. y Salinas, Á. (Eds.), El libro abierto de la informática educativa. Lecciones y desafíos de la Red Enlaces. Santiago: Enlaces- LOM. (2010).

Estévez E., Valdés, A., Arreola., C., y Zavala, M., Creencias sobre Enseñanza y Aprendizaje en Docentes Universitarios Magis, doi: https://doi.org/10.11144/Javeriana.M6-13.CSEA. Revista Internacional de Investigación en Educación, 6(13), 49-64, (2014).

Ertmer, P., Teacher Pedagogical Beliefs: the Final Frontier in our Quest for Technology Integration, Educational Technology, Research and development, 53(4), 25-40, (2005).

Ertmer, P. A., Ottenbreit-Leftwich, A.T., y otros 3 autores, Teacher Beliefs and Technology Integration Practices: A Critical Relationship, doi: https://doi.org/10.1016/j.compedu.2012.02.001. Computers \& Education, 59(2), 423-435, (2012).

Ertmer, P., Anne, O.L., y Tondeur, J., Teacher Beliefs and Uses of Technology to Support 21st Century Teaching and Learning. International handbook of research on teachers' beliefs, 403-419, Routledge, New York, NY, USA, (2015).

European Commissión. European Framework for the Digital Competence of Educators: DigCompEdu. Punie, Y. (ed). EUR 28775 EN. Publications Office of the European Union, Luxembourg, (2017).

Fariña, E., González, C. S., y Area, M., ¿Qué Uso Hacen de Las Aulas Virtuales Los Docentes Universitarios? RED, Revista de Educación a Distancia, 35, (2013).

García, L., Azcarate, C., y Moreno, M., Creencias, Concepciones y Conocimiento professional de Profesores que Enseñan Cálculo Diferencial a Estudiantes de Ciencias Económicas. Relime, 9(1), 85-116, (2006).

García-Chitiva, M., y Suárez-Guerrero, C., Estudio Bibliométrico de la Producción Científica en Web of Science: Formación Profesional y Blended Learning, doi: https://doi.org/10.12795/pixelbit.2019.i56.09, Pixel-Bit. Revista de medios y Educación, 56, 169-191, (2019).

Gramp, J., Beyond the Baseline: Working With e-learning Champions to Transform e-learning at a Research-led University. Second Moodle Research Conference, 24-32, (2013).

Gonzalez, D., Ambientes Colaborativos Virtuales para el Aprendizaje Individual. Revista Actualidades Investigativas en Educación, 17(2) 1-29, (2017). 
Hashim, N., y Jones, M., Activity Theory: A Framework for Qualitative Analysis, This conference paper originally published as Hashim, N and Jones, ML, 4th International Qualitative Research Convention (QRC), 3-5 September, 2007, PJ Hilton, Malaysia. (2017).

Hernández S., El Modelo Constructivista con las Nuevas Tecnologías: Aplicado en el Proceso de Aprendizaje. En: Comunicación y Construcción del Conocimiento en el Nuevo Espacio Tecnológico [monográfico en línea]. Revista de Universidad y Sociedad del Conocimiento (RUSC). Vol. 5, n.ำ 2. UOC, (2008).

INTEF., Marco de Competencia Digital. Madrid: Ministerio de Educación, Cultura y Deportes. (2017).

Jimoyiannis, A., y Komis, V., Teachers Development: An Internacional Journal of Teachers Profesional Development, (2014).

Liberona, D., y Fuenzalida, D., Use of Moodle Platforms in Higher Education: A Chilean Case, doi: https://doi.org/10.1007/978-3-319-10671-7_12. In Communications in Computer and Information Science (Vol. 446 CCIS, pp. 124-134, (2014).

Lašáková, A., Bajzíková , L., y Dedze, I., Barriers and Drivers of Innovation in Higher Education: Case study-based evidence across ten European universities, doi: https://doi.org/10.1016/j.ijedudev.2017.06.002. International Journal of Educational Development, 55(May), 69-79. (2017).

Liu, S-H., C-H. Lin, \& K. K. Zhang, Pedagogical Beliefs and Attitudes Toward Information and Communication Technology: a Survey of Teachers of English as a Foreign Language in China, doi:10.1080/09588221.2017.1347572. Computer Assisted Language Learning, 30(8), 745-765, (2017).

López, L., López, B., y Prieto, E., Tendencias Innovadoras en la Formación on-line, doi: http://dx.doi.org/10.12795/pixelbit.2018. La oferta web de postgrados e-learning y blendedlearning en España, 53, 93-107, (2018).

Mackeogh, K., y Fox, S., Strategies for Embedding e-learning in Traditional Universities: Drivers and Barrier. Electronic Journal of E-Learning Volume, 7(2), 147-154, (2009).

Mama, M., y Hennessy, S., Developing a Typology of Teacher Beliefs and Practices Concerning Classroom use of ICT, Computers \& Education, 68, 380-387, (2013).

Ministerio de Educación de Chile, Estándares en Tecnología de la Información y la Comunicación para la Formación Inicial Docente, Santiago de Chile, Ministerio de Educación, (2006).

Ministerio de Educación de Chile, Estándares en TIC para la Formación Inicial Docente, una Propuesta en el Contexto Chileno, Santiago de Chile, Ministerio de Educación, (2008).

Ministerio de Educación de Chile, Centro de Innovación educativa, (2020).

Ministerio de Educación de Chile, Recomendaciones para un apolítica Digital en educación Escolar (2017)

Orlando, J., Understanding Changes in Teachers' ICT Practices: A Longitudinal Perspective. Technology, Pedagogy and Education, 18(1), 33-44, (2009).

Orlando, J., ICT-Mediated Practice and Constructivist Practices: ¿Is this Still the Best Plan for Teachers' uses of ICT? Technology, Pedagogy and Education, 22, 231-246, (2013).

Pajares, M. F., Teachers Beliefs and Educational Research: Cleaning up a Messy Construct, Review of Educational Research, Vol, 62(3), 307-332, (1992).

Palos, M., Avalos, M. L., Flores, F., y Montes, R., Creencias de Madres y Docentes sobre el Aprendizaje de la Lectoescritura en Educación Preescolar, doi: http://dx.doi.org/10.15517/aie.v17i3.29066. Revista Actualidades Investigativas en Educación, 17(3), 168-189. (2017).

Pérez-Berenguer, D., y Molina, J.G., Un Enfoque para la Creación de Contenido Online Interactivo, doi: http://dx.doi.org/10.6018/red/51/3 Revista de Educación a Distancia, (51), (2016).

Prestridge, S., The Beliefs Behind the Teacher that Influences their ICT Practices. Computers \& Education, 58, 449-458, (2012).

Sánchez, J., Sanchez, P., y Ramos, F., Usos Pedagógicos de Moodle en la Docencia Universitaria desde la Perspectiva de los Estudiantes. Revista Iberoamericana de Educación, 60(60), 15-38. (2012).

Vásquez, M., Aplicación de Modelo Pedagógico Blended Learning en Educación Superior Revista Didáctica, Innovación y Multimedia DIM, año 14, 35, (2017).

Yong-Mi, K., School of Library and Information Studies, University of Oklahoma, Tulsa, Oklahoma, Volume: 36 issue: 5 , page(s): 603-617, (2010). 